\title{
From Digital to Physical: Advancing Industrialized Construction through Digital Prototyping Processes
}

\author{
Basem EID MOHAMED ${ }^{1 *}$, Frederic GEMME ${ }^{2}$, Aaron SPRECHER ${ }^{3}$ \\ ${ }^{1,3}$ McGill University School of Architecture, Montreal, Quebec \\ ${ }^{2}$ BONE Structure ${ }^{\circledR} \mid$ Simple Concept, Laval, Quebec \\ *Corresponding author's e-mail: bassem.eid@mail.mcgill.ca
}

\begin{abstract}
Recent development in digital design strategies and fabrication tools has offered the Architecture, Engineering, and Construction (AEC) industry the opportunity to rethink the prefabricated building industry. Different methodologies have been proposed with the aim of formulating an efficient link between design and fabrication processes, based on devising effective data workflows to overcome complexities associated with conventional production models. This paper demonstrates recent developments in implementing a Digital Prototyping model toward establishing a comprehensive strategy for design and fabrication of a family of building components. The proposed model is specifically designed and implemented to realize a prefabricated construction system, BONE Structure ${ }^{\circledR}$, with the aim of enabling high precision in design and fabrication, in addition to supporting pre-defined assembly on jobsites. This paper represents a phase from an ongoing research endeavor toward infusing the design and production processes with digital logic, aiming for high flexibility and customization in building design, specifically the housing realm.
\end{abstract}

KEYWORDS: Digital Prototyping; industrialized construction; prefabrication

\section{INTRODUCTION}

Industrialization of construction processes emerged along with development in manufacturing methods. Specifically in the early twentieth century, as a result of automobile manufacturer Henry Ford's "serial production" model, architects began questioning the idea of technology transfer from automotive industry to buildings. Ford's method was based on the manufacturing of large standardized components and the systematization of production processes, thus improving on existing methods of sequential production. Systematic repetition of components and processes lowered costs through economies of scale. In that sense, the most notable outcome was the continuous flow of the mass production of Ford's product, making the Model T a remarkably affordable car (Giles 2005).

As many sectors of the market at that time initiated the shift towards mass production models, architects and builders sought to understand why factory production has revolutionized the creation of formerly hand-crafted objects, as well as modern mobility, such as automobiles, while the building industry has been largely resisting the shift toward mass production. Such an enquiry informed the work of many architects; Le Corbusier, Walter Gropius, Frank Lloyd Wright, Bukminster Fuller, and Jean Prouvé; resulting in a wide field of notable experimentations in mass production techniques and their implementation (Kieran and Timberlake 2004). 
During the post-World War II housing boom, industrialization in construction became a necessity, being viewed as a logical solution in response to high demand for housing. Builders took advantage of applying industrial methods based on economic motives, and quality control, targeting the provision of mass housing. Later on, while lagging in the housing industry, the viability of mass production techniques rose and fell in other economic sectors over time. By the 1970s, following a further sharp increase in demand for personalized goods and products, coupled with decline and obsolescence of the standard mass production paradigm, new production model was introduced primarily by Toyota in the automotive industry. First known as the Toyota Production System (TPS), this method became broadly referred to as Lean Production by the 1990s. As a result of further advancements in manufacturing technologies, this new paradigm was received enthusiastically, leading to a new definition, referred to as mass customization, then formally systematized by Joseph Pine in 1993 (Schodek et al. 2005).

Driven by lack of variation and individual personalization in mass production techniques, the building realm has always attempted to adopt knowledge and techniques applied in other sectors to enable new participatory approaches in design. While this interest has taken many forms and constituencies, digital design and manufacturing strategies have inspired the most diverse research and pragmatic solutions to contemporary industry challenges, with the aim of enhancing quality, lowering cost, as well as responding to users' needs and demands.

This paper presents recent developments in implementing a Digital Prototyping model towards establishing an integrated process for design, fabrication, and assembly of a comprehensive construction system; the BONE Structure ${ }^{\circledR}$ system. Based on utilizing an advanced software environment, design and fabrication processes are achieved with significant accuracy, leading to subsequent precision in assembly. Such an application aims at exploring new territories in industrialized construction, toward managing complexities and leveraging quality. The paper represents a phase from an ongoing research endeavor that aims enabling high flexibility in housing design through digitization of the design and production processes.

\section{THE STRUCTURAL SYSTEM: BONE STRUCTURE}

The BONE Structure is a proprietary construction system that combines cold-formed steel components and insulating materials in an integrated manner. The steel components are all designed to be assembled using solely screws and bolts, eliminating the need for cutting, piercing and welding on jobsite. As a consequence, the amount of residual matter resulting from the construction process is reduced significantly. The system differentiates itself from traditional steel construction system by combining the advantages of a post-and-beam structure; larger spans, size of openings, the integration of the structural system with the thermal envelope, and the precision of assembly provided by pre-manufactured components.

Pertaining to form, the BONE Structure is an orthogonal system that has been designed using a 5 feet incremental module in the $\mathrm{X}-\mathrm{Y}$ plane and 6 inches module in the $\mathrm{Z}$ direction, leading high level of standardization of components. Given that the system is always undergoing research and development, the latest version of the system (10.0) includes the half-grid feature, providing more flexibility to the architectural design. 
All components have an overall length of maximum ten feet in order to facilitate procurement. Structural columns have a composite square profile, 4 inches $\mathrm{x} 4$ inches, assembled in plant employing self-tapping screws. An additional $\mathrm{H}$-shaped profile can be added on the exterior side of columns to enhance their resistance to lateral loads. Stronger members are designed using a thicker material whenever required. Figure 1 represents views of the structural system components.
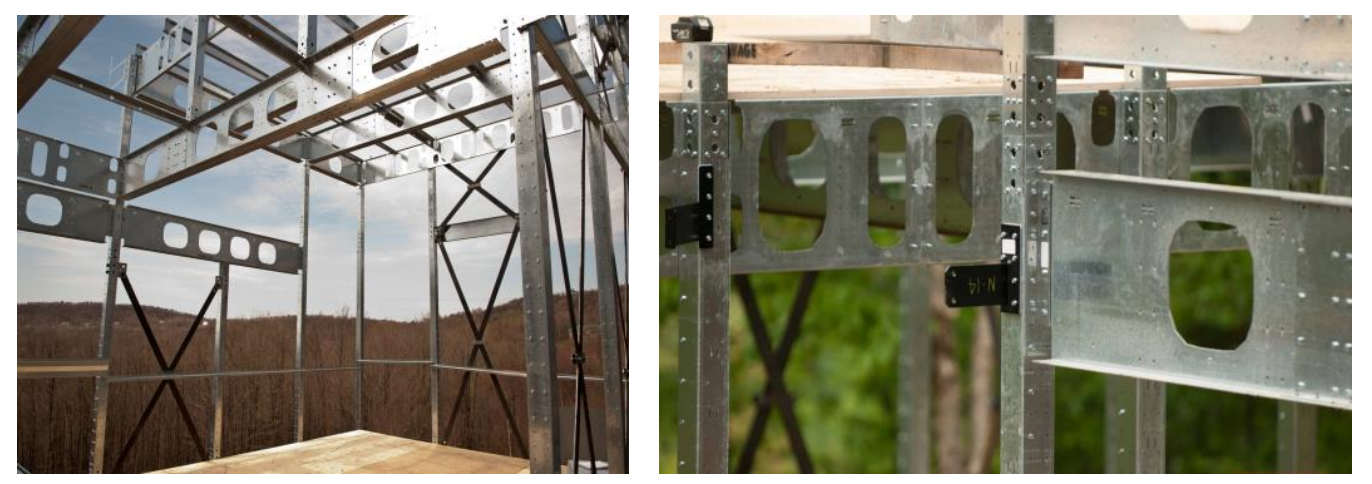

Figure 1: An overview of the structural system, and a close-up for one of the connections.

Floor and roof structure main components are variable lengths of a seventeen inches deep profile manufactured using eleven gauge galvanized steel, the maximum standard span being 25 feet. These profiles are characterized by large openings that are performed to remove some weight to the components and to allow for better efficiency for trades such as electricity, HVAC and plumbing. Stronger members are designed using a thickness of 0.1875 inch when required. Secondary joists are put in the transverse direction between the joists with a typical spacing of 20 inches.

Once land preparation is completed, and the foundation is poured and cured, steel components and fasteners are delivered. The prefabricated structural components are anchored to the foundation by using cast-in anchors available in three standardized dimensions. The anchors are positioned using spacing templates, eliminating the use of a measuring tape. Assembly instructions are obtained from digital models, thus capitalizing on the efficiency of building processes.

Floor structure is completed by adding a 0.75 in thick thirteen plies plywood panel over the steel components. These panels are pre-pierced and pre-cut using CNC (Computer Numerical Control) machining centres. The assembly is performed using self-tapping screws as holes in plywood panels are aligned with holes in the steel structure underneath. The plywood panels are supported on all edges and define a structural diaphragm. On the roof, the diaphragm is achieved by a Structural Insulated Panel (SIP) fastened to the structure by screws installed from the interior side. These SIPs are composed of two layers of Oriented Strand Board (OSB) with an Expensed Polystyrene (EPS) layer in between. The typical thickness of EPS is 10.5 inches, giving an insulating rating of R-40. In some cases, additional structural components such as structural lintels above sliding doors and garage doors, overhangs, balconies, terrace structures and canopies are then added. Openings for doors and windows are pre-framed using pre-cut plywood parts with machined holes and grooves.

The subsequent steps are denoted with covering the complete wall area with 3 inches thick EPS panels. These panels are cut in three configurations to fit exactly in between the structural 
components. They are held in place by $\mathrm{z}$ shaped profiles. The whole wall is finally covered with a 2.5 inches thick layer of polyurethane sprayed foam. The depth of the z profiles is calibrated to exceed the polyurethane and receive the exterior finish. A nylon thermal break positioned between the $\mathrm{z}$ shaped profile and the structural columns prevents metallic continuity through the thermal shell. On the interior, wall and ceilings are covered with galvanized steel furring profiles that are clipped to the structure, making it ready for drywall installation. Exposed components such as architectural stair structure, exposed cross bracings, terrace structure and sunshades are typically installed when spray foam application is completed. The shell is then completed by installing doors and windows and sealing the perimeters of these components.

The presented structural system allows for flexibility in building design through modularity, in addition to specific physical characteristics of its components. This would enable for numerous configuration possibilities, with the aim of responding to users' demand for internal and external design variations.

\section{DIGITAL PROTOTYPING: TOWARDS AN INTEGRATED PROCESS}

Based on previous reviews, the common definition of industrialization in the building industry comprises the concept of manufacturing components within a controlled environment (on or off site), that would then be transported, positioned, then assembled into a structure with minimal site intervention. This includes prefabrication, standardization, automation, and mechanization (Zabihi et al. 2013). Recently, with the significant rising role of design and fabrication technologies, new paradigms have evolved, allowing for better control over the process. Designers and engineers are developing abilities to manage data, simulate structural performance, components production and assembly, towards a more integrated model for construction (Kolarevic 2003) (Schodek et al. 2005).

One of the emerging methods of computer modelling is Digital Prototyping; defined as the mean by which engineers, and designers can explore products virtually before being built. It is a process that allows for designers to visualize, validate, simulate, and optimize, products data throughout the product development process within an advanced a digital environment. Such a process is structured on different levels of automation, depending on the nature of the product, and operations involved in the process (Bullinger et al. 2000).

Digital Prototyping combines Computer Aided Design (CAD) technologies with Virtual Reality (VR) to empower producing prototypes more efficiently, thus optimizing the product development process. One of the strategic advantages of Digital Prototyping is real-time decision making at early stages of product development. This enhances the process and makes it more efficient, leading to allowing for earlier modifications, and optimization of the prototype. Furthermore, virtual prototypes enable qualitative evaluation of product qualities, thus eliminating errors that might occur within the fabrication process.

Bullinger et al. (2000) defined a crucial component of Digital Prototyping that is the Digital MockUp (DMU); a purely digital test model of a technical product. Such a model enables for a current and consistent availability of multiple view of the product shape, function, and technological coherences. This constitutes the basis on which modeling and simulation performed and communicated for an improved configuration of the product. Commonly, virtual products are 
employed as a reference for testing the design regarding its feasibility, functionality, and efficiency prior to production of physical prototypes. This is done by building a comprehensive model that brings 3D data into a single 3D model. This is done either for exclusive products, or ones integrated within a system. In such a virtual environment, possible defects can be detected and corrected in design before building physical products. The model also enables unprecedented precision in establishing the link between design and manufacturing, thus improving productivity.

Digital Prototyping as an approach has been successfully implemented in various domains, such as aircraft construction, shipbuilding, and the automotive industry. Also, various software platforms have been employed, including for instance, Autodesk Inventor, Solidworks, and CATIA by Dassault. Nevertheless, the selection criteria of the platform relates to the capability of devising a workflow that is compatible with the nature of the product, level of technology involved, and users participating in the product development process. We present in this research a workflow structure that complements the nature of the construction system, as well as the targeted flexibility strategy, by enabling interoperability for efficient data exchange throughout the design, production, and assembly processes.

\section{Digital Prototyping in design}

The BONE Structure technology constitutes an ensemble of components that are designed, and validated through Digital Prototyping tools. Commonly, the building design process is initiated through a conceptual framework. Once further elaborated, the conceptual framework is then implemented within a Building Information Modeling (BIM) environment; Autodesk Revit, aiming for a comprehensive 3-Dimentional object representing the building. The modeling process employs a set of pre-designed components stored within a database of families, thus becoming a process of virtual assembly.

The structure of the utilized design and modeling environment is well adapted to the standardization of BONE Structure's technology, where the BIM families' database comprises detailed models of structural columns and joists, in addition to walls, floors, roofs, openings, stairs, exterior canopies and balconies. These components have been parametrically designed in order to provide flexibility while keeping the design possibilities within a feasible range. Typically, this type of BIM-based virtual assembly aims at achieving the following:

- Develop, and validate the architectural design;

- Export the structural components for advanced structural analysis;

- Provide referential information for digital prototyping of individual components.

While BIM allows for proper visualization to communicate with clients, it also minimizes technical conflicts throughout the design significantly, where technical components are updated in real-time, in accordance with design decisions.

Pertaining to structural design, analysis is performed by first building a 3D model of linear members with predefined geometrical and mechanical properties. These members are then virtually simulated to environmental loads based contextual conditions, and building codes. Reactions on members and connexions are computed using numerical methods, enabling for precise definition of appropriate profiles to withstand the calculated loads. The implementation of a BIM environment allows for efficient interoperability between architectural modeling and structural analysis, whereas required structural modifications are automatically applied to the BIM 
model, resulting in significant time savings. Once validated, the outcome of this process is then transferred to the Digital Prototyping platform, where building foundation, exterior doors, windows and roof in a non-editable format, are used to provide reference levels and size of interfacial elements.

While there are various approaches applied in the industry for design and modeling, the distinctive approach of BONE Structure to Digital Prototyping of building components stems from critical analysis of Product Lifecycle Management (PLM) principles applied in other industries such as aerospace and automotive. Using Autodesk Inventor software, referential information obtained from the BIM model allows for standard components to be selected from a components' library and assembled following predefined principles. Indeed, the virtual assembly is done in the following sequence:

- Primary structure: automated placement of structural components such as columns and beams using an algorithm that was designed and coded specifically for such an operation. The algorithm places perimeter components, then defines joist layout for the following step;

- Exterior doors and windows: pre-frames are added using a second algorithm that delineates all individual parts from the basic window dimensions;

- Exterior and interior finish sub-frames: Supports are added to the 3D model using a third algorithm that automates positioning around openings;

- Roof panels: overall dimensions are defined by the user and individual panel layout is done automatically;

- Stairs: regarded as a custom part, parametric logic is used to enter specific design parameters in a predefined model;

- Custom parts: some architectural elements may require the design of new parts which is done directly within the three-dimensional assembly of components, securing the conformity of the part within the assembly.

The outcome of this process becomes a comprehensive 3D model, comprising adequate information required to proceed to fabrication of the various components. Figure 2 represents a print screen of structural components assembly on the Digital Prototyping platform.
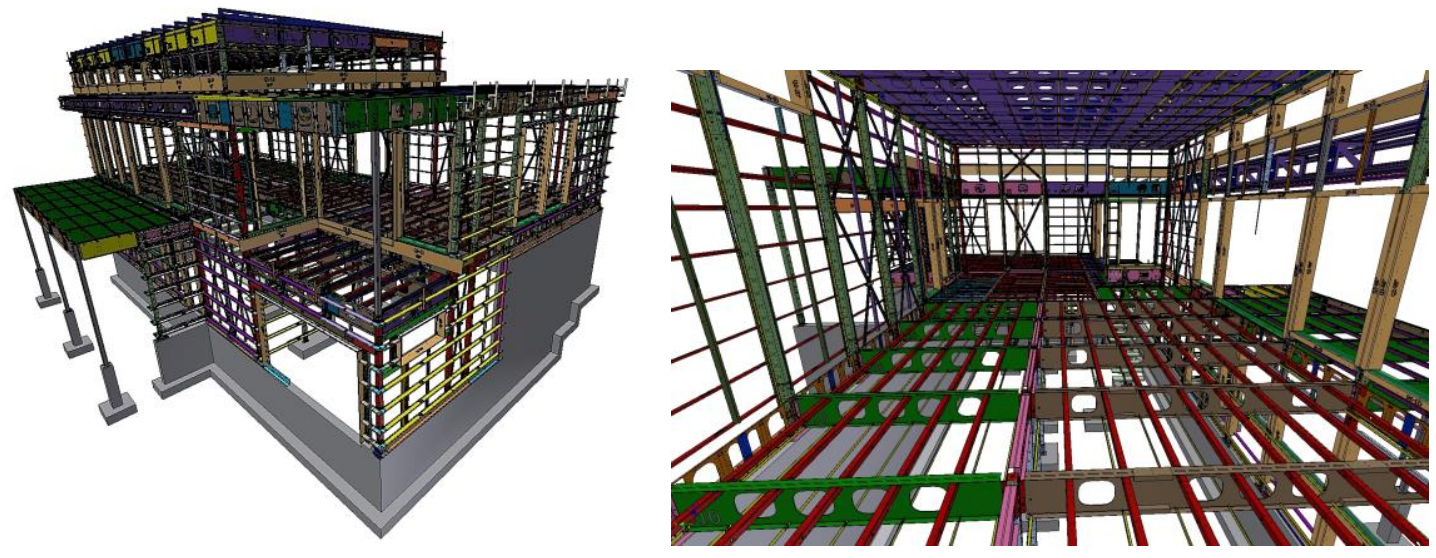

Figure 2: A screen-shot from Autodesk Inventor of a 3D model demonstrating levels of details in components and connections employing fully standardized parts. 


\section{Digital Prototyping towards fabrication and assembly}

The implemented Digital Prototyping strategy supports a multi-level digital design environment that benefits abundantly from Computer Aided Design/Computer Aided Manufacturing (CAD/CAM) processes. It is structured based on a set of modules devised to aid in the process of modeling, simulation, fabrication, and assembly of components for the BONE Structure system. Commonly, a BONE Structure residential home project would include between 200 to 400 components in variable quantities, for a total in the range of 8000 to 15000 individual steel parts. These components are divided into twelve families, based on the type of material and manufacturing process.

Pertaining to fabrication, given that the system implies eliminating measurements, cutting, and welding on site, yet only screwed and bolted connections, production relies on an effective fileto-factory process. Fabrication instructions are extracted from 3D models, then sent for fabrication via digital files, including bills of materials. These instruction are used to operate CNC machines, such as punch presses, laser cutting, bending and stamping, for the fabrication of various components. Additionally, robotized welding is used for complex assemblies.

Once fabrication is completed, the assembly process is initiated two phases: pre-assembly infactory and on-site assembly. In parallel with fabrication progression, the engineering team works on pre-assembly instructions, thus documenting quantities of families and hardware components, steel parts, fasteners, as well as precise sequential assembly procedures (Figure 3). Accordingly, the process becomes a predefined systematic practice that leverages quality, saves time, and minimize waste remarkably, toward a sustainable model.
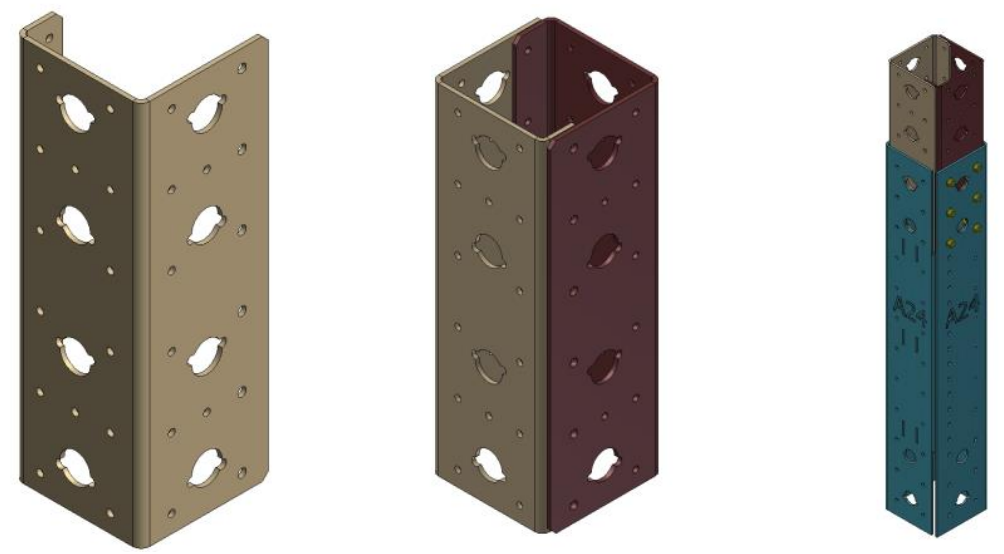

Figure 3: A screen-shot from Autodesk Inventor of a 3D model of a components and its assembly model prior to site delivery.

Based on the same virtual model, on-site assembly instructions are also generated to provide jobsite team with precise instructions. These instructions are provided in two formats. First, colorcoded three-dimensional drawings, comprising symbols, part number, notes, and sections (Figure 4). On the other hand, cloud-based 3D visualization interfaces is utilized to assist assembly team throughout the building process.

The application of digital tools aims at providing the means for managing and exchanging data during the design, fabrication, assembly, and construction processes. The proposed model is devised based on analysing available software platforms, while focusing on efficient 
interoperability, ease of use, and technical support. Nevertheless, maintaining such qualities requires continuous Research and Development activities by the team in order to keep up with pace of software developments, thus informing the process constantly.
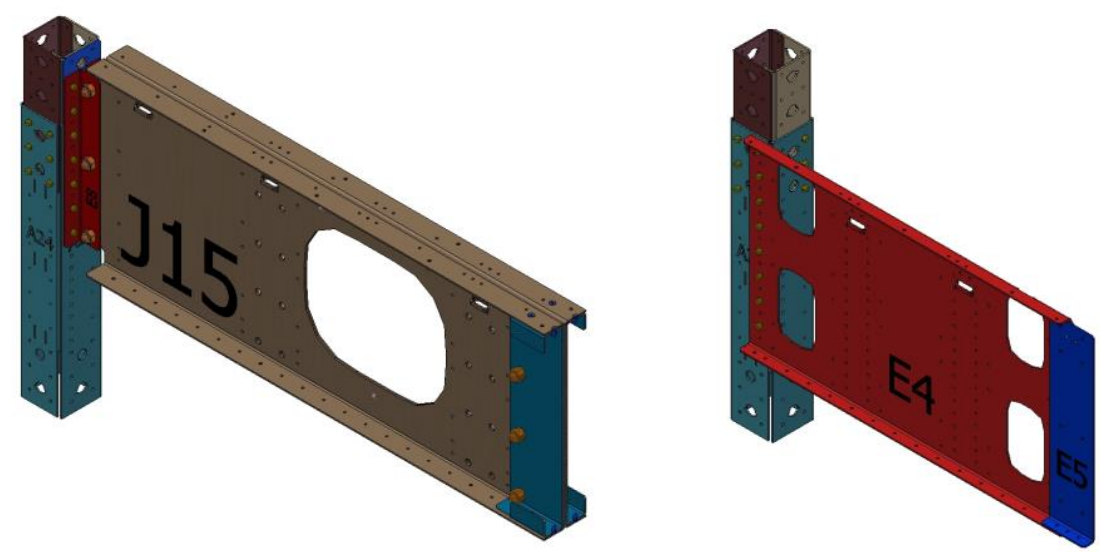

Figure 4: A screen-shot from Autodesk Inventor of a 3D model of a components and its assembly instructions for jobsite.

\section{CONCLUSION}

Recent developments in design and fabrication technologies have opened new opportunities that can be considered as a leading edge of a chain of innovation in the building industry. While industrialized construction have witnessed great interest as an approach, digital design and manufacturing strategies have inspired the most diverse research and pragmatic solutions to contemporary industry challenges. Nevertheless, the influence of these technologies only becomes effective when core systems are imbedded within a collaborative platform of interoperability. In such a case, different participants ultimately exchange data, and contribute towards an integrated process; design, analysis, simulation, fabrication, and assembly.

This paper represents a technological approach to advance industrialized construction practices, through an integrated strategy. The proposed strategy combines a state-of-the-art construction system, and a digital modeling platform; Digital Prototyping. Whereas Digital Prototyping has been applied in many engineering and design domains, yet in architecture, such an approach is still in an exploratory phase. The described Digital Prototyping platform in this paper tends to complement the tactility of the construction system to leverage quality and design flexibility. This relates directly to the concept of data management and collaborative abilities, leading to effective communication between design team members, as well as fabricators. Furthermore, to surpass conventional capabilities of the platform, computational design logic in the form of a placement algorithms has been coded, and implemented specifically to enhance digital processes. These computational tactics capitalize on the structural integrity of the construction system, through automating a set of instruction within the design and modeling process.

The work described in this paper represents a phase from an ongoing endeavor towards enabling high level of design flexibility towards mass customization in the realm of architecture. The proposed integrated approach, in the form of combining a structural system, and a technological strategy, is seen as a crucial element to realize the paradigm of mass customization, specifically in 
the housing realm. Future exploration will focus on integrating computational BIM, and real-time environmental analysis within the design process.

\section{REFERENCES}

Bullinger, H.-J, Warschat j., and D. Fischer. (2000). "Rapid product development — an overview." Computers in Industry, 42 (2-3):99-108.

Giles, H. (2008). "Prefabricated Construction using Digitally Integrated Industrial Manufacturing" ARCC Journal of Architectural Research, 5 (2):48-65.

Kanai,S., Taiga, H., and Kikuta, Y. (2009). "3D digital prototyping and usability enhancement of information appliances based on UsiXML." International Journal on Interactive Design and Manufacturing (IJIDeM), 3 (3):201-222.

Kolarevic, B. (2003). Architecture in the digital age : design and manufacturing, Spon Press, New York, NY.

Kieran, S., and Timberlake, J. (2003). refabricating ARCHITECTURE: How Manufacturing Methodologies are Poised to Transform Building Construction. McGraw-Hill Professional, NY.

Petric, J., and Maver, T. (2003). "Digital prototyping in the architectural design studio." SIGRADI, Rosario, 29-38.

Pine, B J. (1993). Mass customization : the new frontier in business competition, Harvard Business School Press, Boston, Mass.

Schodek, D., Bechthold, M., Kimo,J., Kenneth, G.,, and Steinberg M. (2005). Digital Design and Manufacturing: CAD/CAM Applications in Architecture and Design, John Wiley \& Sons, New Jersey

Toffler, A. (1970). Future Shock. Random House, New York.

Toffler, A. (1980). The third wave, Morrow, New York .

Tseng, Mitchell M., and Frank T Piller. 2003. The customer centric enterprise : advances in mass customization and personalizaton, Springer, New York.

Zahibi, H., Habib, F., and Mirsaeedi,L. (2013). "Definitions, Concepts and New Directions in Industrialized Building Systems (IBS)" KSCE Journal of Civil Engineering, 17(6):1199-1205. 